\title{
切削工况下机床主轴回转精度动态预测方法*
}

康 婷 1 曹宏瑞 ${ }^{1,2}$

(1. 西安交通大学机械工程学院 西安 710049;

2. 西安交通大学机械制造系统工程国家重点实验室 西安 710049)

\begin{abstract}
摘要: 回转精度是衡量主轴加工性能的重要指标。现有的回转精度测量方法通常借助标准球并在空转条件下进行, 无法计入 切削载荷等负载带来的影响, 难以反映主轴在切削状态下的真实精度。针对该问题, 提出一种基于动力学模型的主轴回转精 度动态预测方法。首先建立高保真的高速主轴-轴承系统动力学模型, 并将切削力等激励载荷作为输入边界条件。然后利用测 力仪测量、修正切削力, 并将其输入动力学模型, 实现主轴振动响应和径向回转误差的动态预测。设计并制作一套主轴回转 误差在线测量装置, 对回转精度预测结果进行试验验证。将不同切削工况下的仿真与试验结果进行对比分析, 结果表明提出 的方法能够准确预测主轴回转精度的变化规律, 可以为主轴加工性能评估提供依据。
\end{abstract}

关键词: 回转精度; 机床主轴; 动力学建模; 切削工况; 动态预测

中图分类号: TG659

\section{Dynamic Prediction Method for Machine Tool Spindle Rotational Accuracy under Cutting Condition}

\author{
KANG Ting ${ }^{1}$ CAO Hongrui ${ }^{1,2}$
}

(1. School of Mechanical Engineering, Xi'an Jiaotong University, Xi'an 710049;

2. State Key Laboratory for Manufacturing Systems Engineering, Xi'an Jiaotong University, Xi’an 710049)

\begin{abstract}
Rotational accuracy is an important indicator of spindle machining performance. The existing measurement methods of the rotational accuracy are usually carried out by means of a standard ball and under idling conditions, which cannot take into account the influence of the load such as the cutting load. Therefore the existing methods are difficult to reflect the true accuracy of the spindle in the cutting state. Aiming at this problem, a dynamic prediction method of spindle rotational accuracy based on dynamic model is proposed. Firstly, a high-fidelity high-speed spindle-bearing system dynamics model is established, and the excitation load such as cutting force is taken as the input boundary condition. Then, the cutting force is measured by a dynamometer and modified and input into the dynamic model to realize the dynamic prediction of vibration response and radial rotational error of the spindle. A set of on line measuring device for spindle rotational error is designed and manufactured, and the prediction results of the rotational accuracy are experimentally verified. The simulation and experimental results under different cutting conditions are compared and analyzed. The results show that the proposed method can accurately predict the variation of spindle rotational accuracy and provide a basis for the evaluation of spindle machining performance.
\end{abstract}

Key words: rotational accuracy; machine tool; dynamic modeling; cutting condition; prediction

\section{0 前言}

机床主轴的回转精度直接影响加工零件的形状 误差与表面质量, 是衡量主轴综合性能的重要指标 之一 ${ }^{[1]}$ 。目前, 主轴回转精度的研究热点主要集中

* 国家自然科学基金(51922084)和国家重点研发计划(2018YFB2000502) 资助项目。20190906 收到初稿, 20200319 收到修改稿
在回转精度的形成机理 ${ }^{[2-5]}$ 、回转精度与工件精度的 映射关系 ${ }^{[6-7]}$ 、主轴回转精度的测量方法 ${ }^{[8-10]}$ 、主轴 回转精度控制 ${ }^{[11-13]}$ 几个方面。其中回转精度的测量 方法一直是研究热点。主轴回转精度的测量方法包 括打表测量法、单向和双向测量法、误差分离技术 与 $\mathrm{CCD}$ 成像技术测量法等。打表测量法是早期用 于测量主轴的跳动值近似当做回转精度, 误差大, 而且是静态或极低转速下的状态，目前很难用于测 量精密主轴的回转精度; 单向和双向测量法简单方 
便, 主要针对精度不高的主轴, 大多是离线或在位 测量, 忽略了测量面的形状误差 ${ }^{[14]}$ 。而误差分离技 术与 CCD 成像技术测量法只针对当前精密超精密 主轴回转精度的测量, 测量精度可达亚微米甚至纳 米级别 ${ }^{[15]}$ 。CCD 成像技术法设备成本高、技术复杂, 实际中很少使用; 基于误差分离技术的主轴回转精 度测量方法可分离出测量面的形状误差(圆度误差) 和回转运动误差, 广泛用于高精密主轴回转精度的 测量。误差分离技术测量主轴回转精度常用的方法 有: 反转法、多步法和多点法三种。反转法和多步 法需要标准件在主轴不同角度位置多次连续测量, 因此, 出现了两个相关的误差源: 标准球的形状误 差和回转运动误差的不重复性, 并且这两种方法无 法实现在线测量。虽然三点法可以实现在线测量, 但三点法同样需要借助标准球, 且只能实现主轴空 转条件下的回转精度测试。然而当主轴运行在高速 切削条件时, 切削载荷、转速等边界条件的变化会 引起主轴回转精度的变化, 空转等条件测量得到的 主轴回转精度难以反映主轴在实际加工过程的运行 精度。现有的主轴回转精度测量方法所需步骤复杂, 且对试验装置精度要求高, 难以在线监测主轴在实 际切削过程中的回转精度。建立机床主轴动力学模 型, 并利用模型对回转精度进行动态预测是实现主 轴在切削过程中回转精度评估的有效途径之一。

在过去的几十年里, 学者们针对主轴动力学问 题开展了大量的研究, 建立了动力学模型并开展振 动响应、模态分析及优化设计等研究工作。例如,

KARACAY 等 ${ }^{[16]}$ 利用角接触球轴承支承的刚性转子 磨床主轴动力学模型, 研究了主轴在径向、轴向以 及角度摆动方向的振动; $\mathrm{KIM}$ 等 ${ }^{[17]}$ 建立主轴动力学 模型, 考虑了轴承配合间隙, 研究了结构参数、运 行参数以及热因素对主轴系统的影响; 国内蒋书运 等 ${ }^{[18-20]}$ 建立高速主轴系统动力学模型, 对系统临界 转速、主振型、轴承轴向预紧力等动态特性进行分 析。陈小安等 ${ }^{[21-22]}$ 建立立式主轴模型, 研究了主轴 轴向振动特性、热特性以及对主轴精度的影响。曹 宏瑞等 ${ }^{[23-25]}$ 基于 Gupta 轴承动力学模型和转子有限 元模型建立了主轴动力学模型, 并利用该模型研究 了不同工况下主轴系统的动力学响应。目前多数研 究利用主轴动力学模型研究主轴刚度、固有频率等 动力学参数, 并开展优化设计。由于对滚动轴承动 力学模型做了大量的简化, 鲜有研究对主轴回转精 度进行预测。

本文基于 $\mathrm{XI}$ 等 ${ }^{[25]}$ 建立的高速主轴动力学模型, 提出了切削工况下主轴回转精度预测方法。首先,
建立高速主轴系统动力学模型; 然后, 提出基于高 速主轴动力学模型的回转精度预测方法。最后, 为 了试验验证提出的回转精度预测方法的正确性, 设 计了一套无需借助标准球的主轴回转精度测试装 置, 实现了切削工况下的主轴回转精度测量。仿真 结果与试验具有较好的一致性, 证明了提出的动力 学模型驱动的切削工况下主轴回转精度预测方法的 合理性。

\section{1 高速主轴-轴承系统动力学建模}

主轴的回转运动误差由转子回转特性和轴承承 载特性互相耦合形成 ${ }^{[26]}$ 。因此, 建立准确的主轴转 子模型和轴承动力学模型是准确预测主轴回转精度 的重要前提。同时, 为了使基于动力学模型的回转 精度预测结果与实际回转精度更加接近, 还应建立 主轴刀柄、刀具以及主轴箱的动力学模型。

图 1 为 XI 等 ${ }^{[25]}$ 针对实验室型号为 Kessler DMS 080 的高速主轴建立的主轴动力学模型。该主轴的 最高转速为 $24000 \mathrm{r} / \mathrm{min}$ 。主轴前端由一对角接触 球轴承(FAG HC 71914)支撑, 以背靠背的形式安装, 后端由一个浮动变位轴承(FAG 1011- DLR-T-P4S ) 支承。主轴动力学模型包括主轴转子、轴承、主轴 箱体、刀柄和刀具, 刀具为单刀铣刀。主轴前端的 角接触球轴承和后端的浮动变位轴承均采用 Gupta 轴承动力学建模方法, 能够综合考虑轴承各部件的 三维运动、离心力、陀螺力矩效应、润滑牵引、接 触面打滑以及保持架碰撞等复杂轴承动力学特性。 主轴以弹簧预紧的方式进行预紧, 预紧力施加在前 端角接触球轴承对上。浮动变位轴承主要起到支撑 轴承, 同时实现对主轴轴向热膨胀的补偿作用。主 轴转子动力学模型采用基于 5 自由度 Timoshenko 梁 单元的有限元模型, 可以综合考虑转子弯曲、剪切 变形、转动惯量以及高速下转子离心力和陀螺力矩 的影响。轴承-转子动力学模型之间的耦合约束, 主 要通过轴承内圈与其在转子上相应安装节点之间的 作用力参数和振动响应参数相互传递实现。刀柄和 刀具采用 5 自由度 Timoshenko 梁单元建模, 刀柄主轴以及刀具-刀柄之间均采用刚性连接方式。主轴 箱为 4 自由度刚性转子模型, 与轴承外圈的耦合约 束通过主轴箱上轴承外圈安装节点和轴承外圈之间 的相互作用力传递以及振动响应约束来实现。具体 的转子模型、轴承模型、轴承-转子的耦合方式、主 轴箱模型可查阅文献[25], 不再详细赘述。 


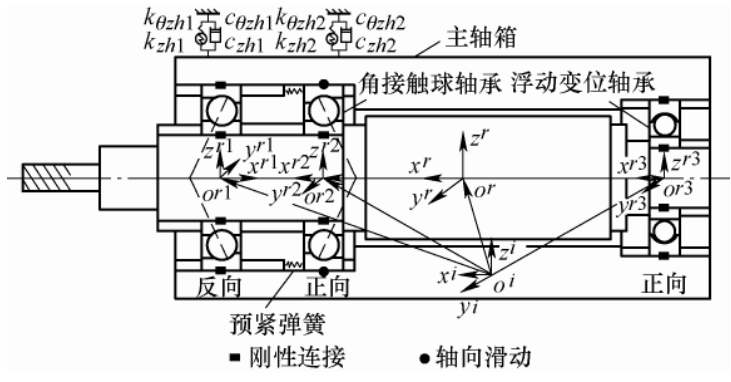

图 1 高速主轴主轴箱-轴承-转子系统结构示意图

\section{2 回转精度预测方法}

对于机床主轴来说, 其回转误差运动可以分解 为: 同步误差运动和异步误差运动。主轴在切削工 况下噪声较大, 噪声会严重影响异步误差的评定。 由于动力学模型无法考虑实际切削过程中的噪声, 因此只说明基于主轴动力学模型的同步误差预测。 图 2 为提出的动力学模型驱动的主轴回转精度预测 方法, 具体步骤如下所述。

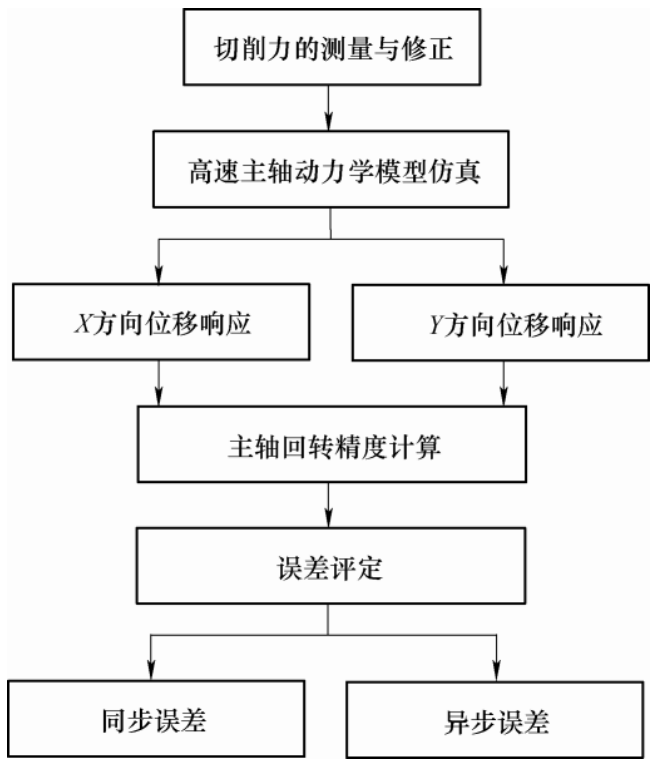

图 2 回转精度预测流程图

(1) 切削力的测量与修正。

通过测力仪获得主轴在切削过程中的实测切削 力, 接着对实测切削力进行修正。通过从测力仪系 统频响函数中提取相关信息, 并结合测力仪系统各 方向固有频率, 利用样条拟合插值方法计算测力仪 在 $X 、 Y 、 Z$ 方向不同切削力频率分量对应的幅值修 正因子。对于给定方向的实测切削力信号, 将切削 力信号傅里叶变换得到的每个离散频率位置处的幅 值(这里是指傅里叶变换得到的复数值)与该方向对 应每个离散频率位置处的切削力幅值修正因子相
乘, 得到修正后真实的切削力频谱。然后, 将修正 后的切削力傅里叶变换频谱经过逆傅里叶变换计算 得到切削过程的真实动态切削力。

切削力的修正是准确预测主轴回转精度的关键 一步。图 3 为主轴转速为 $6000 \mathrm{r} / \mathrm{min}$, 切深为 $1.6 \mathrm{~mm}$, 切宽 $2 \mathrm{~mm}$, 进给为 $500 \mathrm{~mm} / \mathrm{min}$ 工况下 的 $Z$ 方向切削力修正前后时域波形及其频谱的对比 图。从修正后的结果来看, 对于测得的切削力进行 修正是十分必要的。
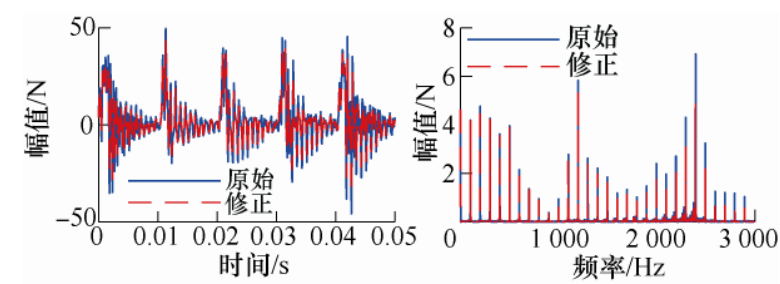

图 3 切削力修正前后对比

(2) 高速主轴动力学模型仿真。

将修正后的切削力信号作用于高速主轴动力学 模型刀尖位置处，仿真得到刀柄相应节点处的振动 位移响应。这里需要注意的是, 主轴动力学模型中 的切削力激励点和振动位移响应测点应与试验保持 一致，如图 4 所示。
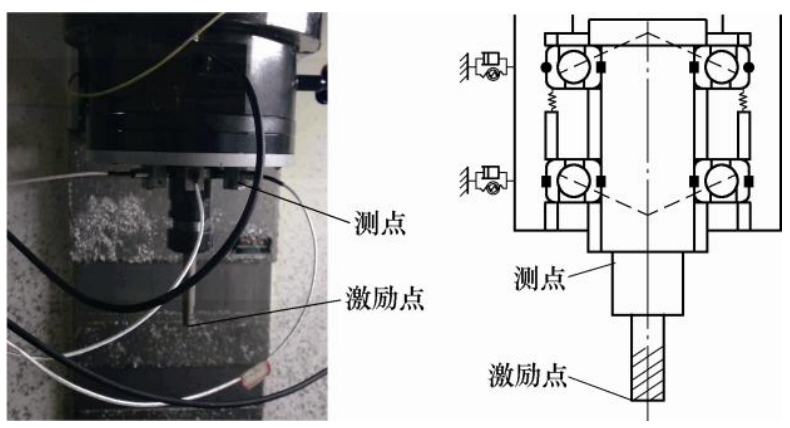

图 4 仿真与试验条件对比图

(3) 主轴回转精度计算。

将仿真得到的主轴 $X$ 和 $Y$ 方向的振动位移信号 经过重采样, 去偏心后代入式(1)计算得到主轴的回 转精度 $E(\theta)$ 。

$$
E(\theta)=\Delta X(\theta) \times \cos \theta+\Delta Y(\theta) \times \sin \theta
$$

(4) 误差评定。

采用基于回转误差运动圆图像的最小二乘圆圆 心法评定回转精度的大小。图 5 是基于误差运动圆 图像的以最小二乘圆圆心法评定误差的示例图。图 中多圈的黑色轨迹就是总误差运动, 绿色曲线就是 总误差运动各个角位置对应的平均值曲线, 即同步 误差运动。LSC(Least-squares circle centre)是同步误 
差运动轨迹点的最小二乘圆圆心, $\mathrm{PC}(\mathrm{Centre}$ of the polar chart) 是误差运动极坐标圆图像的中心点。图 中以 LSC 为圆心的两个蓝色同心圆分别是总误差运 动轨迹的内切和外接圆, 两圆半径差 $a$ 就是总误差 运动的评定值; 同样两个红色同心圆以 LSC 为圆心, 两者半径差 $b$ 就是同步误差运动的评定值; 而同一 角位置处的总误差运动的峰谷值的最大值 $c$ 就是以 $\mathrm{PC}$ 为中心评定的异步误差值。

$$
\begin{array}{ll}
\text { 一 总误差运动 } & a \text { 总误差值 } \\
\text { 一 同步误差运动 } & b \text { 同步误差值 } \\
+ \text { LSC } & c \text { 异步误差值 }
\end{array}
$$

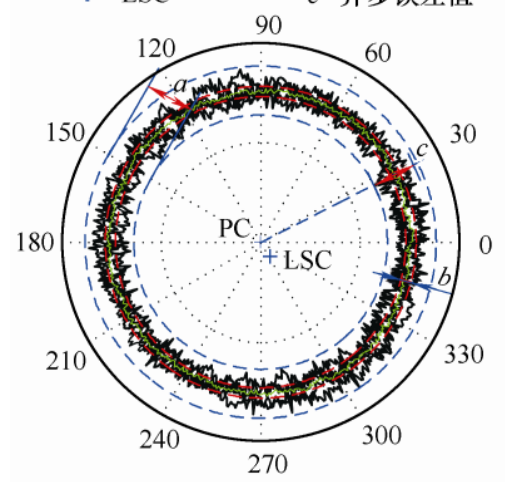

图 5 误差运动圆图像

采用上述误差评定方法对同步误差, 异步误差 以及总误差进行评定, 得到各个误差大小。

\section{3 试验验证}

\section{1 试验设置}

采用三点误差分离法测试主轴的回转精度。三 点法误差分离原理如图 6 所示。

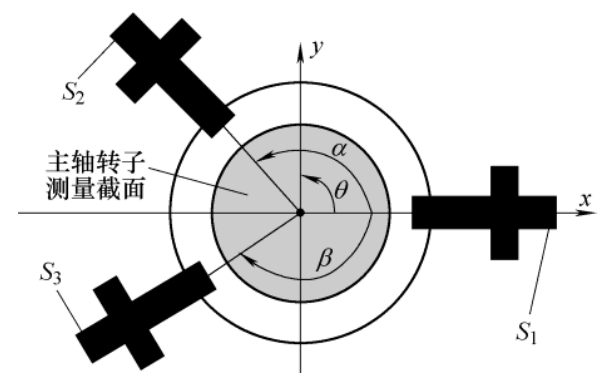

图 6 三点法误差分离示意图

将三个传感器 $S_{1} 、 S_{2} 、 S_{3}$ 安装在主轴圆周测量 截面上。该方法对三个传感器的安装位置要求高, 因此针对 Kessler DMS 080 主轴设计并加工了如 图 7a 所示的分度盘, 该分度盘上存在主尺和游标 尺, 圆形分度盘为主尺, 其上标有间隔 $1^{\circ}$ 的刻度。 扇形传感器探头支座为附尺, 附尺上标有精度为 $0.1^{\circ}$ 的刻度。传感器探头支座上有垂直于主轴轴向的安 装孔, 用于安装位移传感器的探头。该分度盘上共
有三个传感器探头支座, 分别用于安装三个位移传 感器。传感器探头支座压在分度盘上并与支座底座 相连, 支座底座被压板压在底座固定盘的凹槽里, 可在凹槽里滑动。当传感器探头支座的角度调整好 后, 拧紧支座底座螺钉孔里的螺钉可将滑动的支座 底座固定在底座固定盘的凹槽里。由此实现三个传 感器的精确安装和固定。分度盘通过底座固定盘和 联接盘连接到主轴上, 连接方式如图 7b 所示。连接 好后, 传感器探头正对刀柄的高精度测量圆柱面, 此圆柱面代替传统的标准棒, 从而实现切削工况下 的主轴回转精度测量。该装置能够精确确定各个传 感器探头的安装角度, 精确度达到 $0.1^{\circ}$, 可以很大 程度上提高回转误差的测量精度, 为主轴回转误差 与圆度误差的成功分离提供有力的支撑条件。

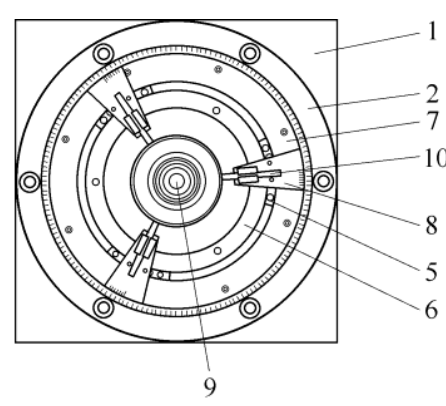

(a) 分度盘

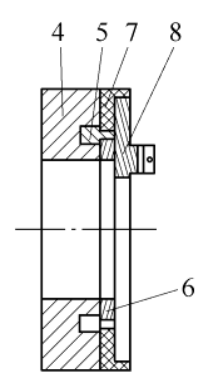

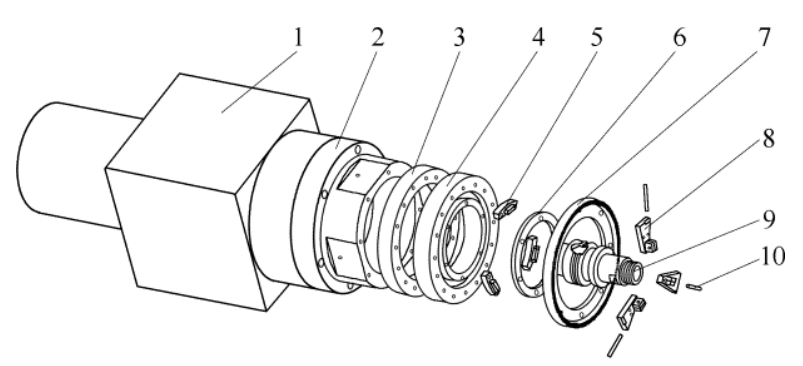

(b) 分度盘与主轴连接方式
图 7 主轴回转精度测量装置

1. 主轴箱体 2. 主轴固定套 3. 联接盘 4. 底座固定盘 5. 支座底座 6. 压板 7. 分度盘 8. 传感器探头支座 9 . 刀柄 10 . 传感器探头

三点误差分离算法要求对各个传感器的信号等 角度采样。一些学者 ${ }^{[27-28]}$ 用等时间采样信号代替等 角度采样信号, 但是如果主轴存在转速波动, 这样 的处理方式会使测量得到的主轴回转精度存在较大 误差。因此, 借助机床主轴编码器信号和 NIPXI5122 采集仪器实现位移信号的等角度采样。主轴编码器 每转产生 512 个等间隔的脉冲, 512 个脉冲触发位 移传感器等角度采集位移信号。

图 8 为基于三点法的切削工况下主轴回转精度 测量试验仪器连接图。测量主轴回转精度的同时使 用奇石乐测力仪测量切削力信号, 切削力信号通过 亿恒数据采集器采集, 送入计算机进行数据存储并 
显示, 切削力信号的采样频率为 $6000 \mathrm{~Hz}$ 。回转精 度测量截面为主轴刀柄位置处一光滑测量面, 在该 截面上分别布置 3 个雄狮位移传感器。使用设计的 分度盘安装并固定传感器, 选取三个传感器的安装 角度为 $\alpha=45^{\circ}, \beta=51.6$ 。位移传感器灵敏度为 $80000 \mathrm{mV} / \mu \mathrm{m}$, 量程为 $0 \sim 250 \mu \mathrm{m}$ 。三个传感器采 集的位移信号先经过信号调理器, 然后经过数据采 集器中处理, 送入计算机进行数据存储。图 9 为回 转精度计算流程图。在该流程图中, 同步平均是降 低位移信号中噪声的关键一步。三点法误差分离算 法可参考文献[27]。将采集到的三个传感器的位移 信号经过图 9 的回转精度计算流程计算得到不同工 况下的回转精度。

\section{传感器 2}

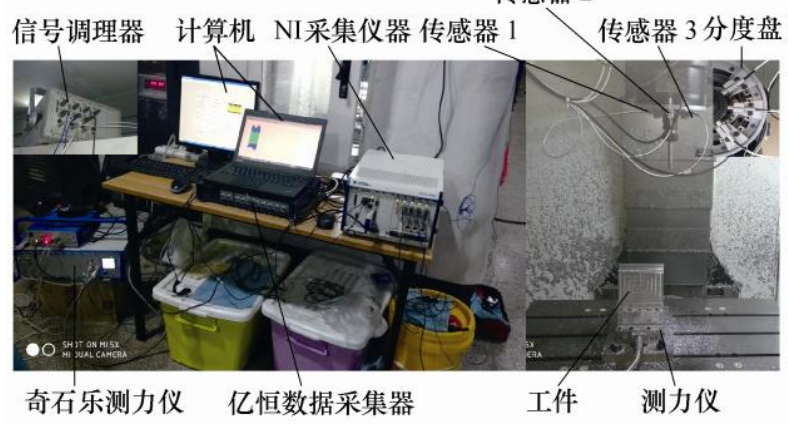

图 8 切削工况下主轴回转精度测量

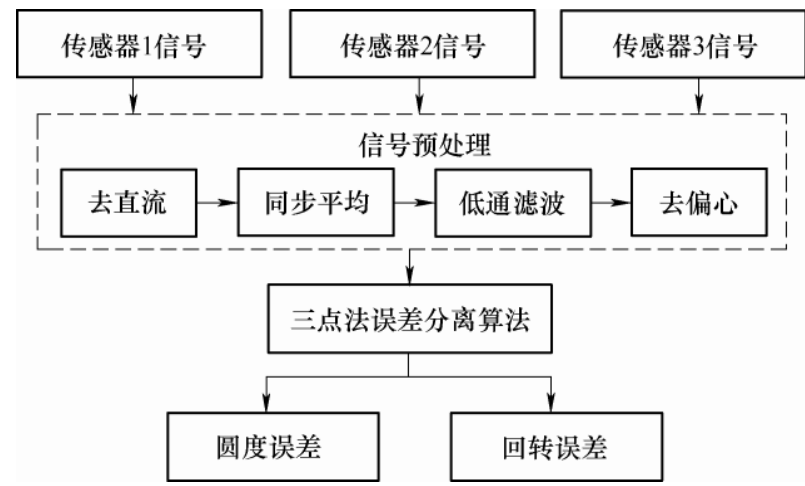

图 9 回转精度计算流程

在主轴分别以 $100 \mathrm{r} / \mathrm{min}$ 和 $500 \mathrm{r} / \mathrm{min}$ 空转条件 下, 采集位移信号, 按照上述计算流程得到两组转 速的圆度误差, 如图 10 所示。从图 10 中可以看到, 两转速下的圆度误差吻合得很好, 说明了上述回转 精度计算方法的准确性。

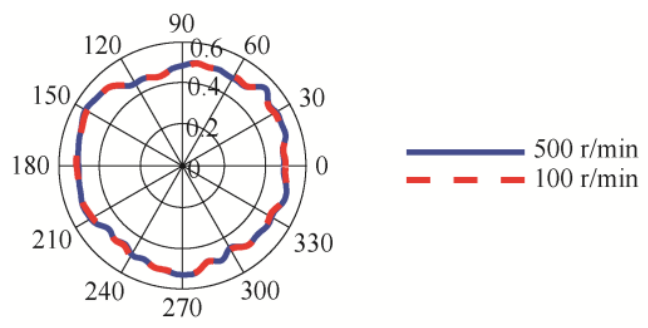

图 10 圆度误差
分别在三种切削工况下测量主轴的回转精度和 切削力, 如表 1 所示。 3 种工况的主轴转速均为 $6000 \mathrm{r} / \mathrm{min}$ 。变进给工况下进给速度每 $200 \mathrm{~mm} / \mathrm{min}$ 变化一次; 变切宽工况下切宽每 $2 \mathrm{~mm}$ 变化一次; 变切深工况下切深每 $0.2 \mathrm{~mm}$ 变化一次。

\section{表 1 回转精度测量工况}

\begin{tabular}{cccc}
\hline 工况 & 进给速度 $/(\mathrm{mm} / \mathrm{min})$ & 切宽 $/ \mathrm{mm}$ & 切深 $/ \mathrm{mm}$ \\
\hline 变进给 & $200 \sim 1200$ & 2 & 0.2 \\
变切宽 & 500 & $1 \sim 12$ & 0.2 \\
变切深 & 500 & 2 & $0.2 \sim 1.6$ \\
\hline
\end{tabular}

将上述工况下测得的三个传感器信号经过图 9 的回转精度计算流程计算得到各个工况下主轴的回 转精度。根据图 5 的回转精度评定方法得到三种工 况下的同步误差、异步误差和总误差，计算结果如 图 11 所示。

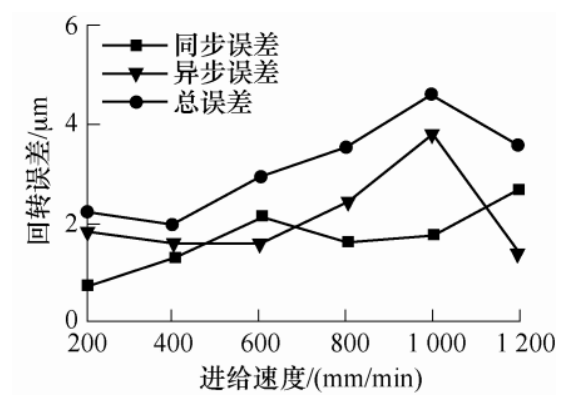

(a) 变进给

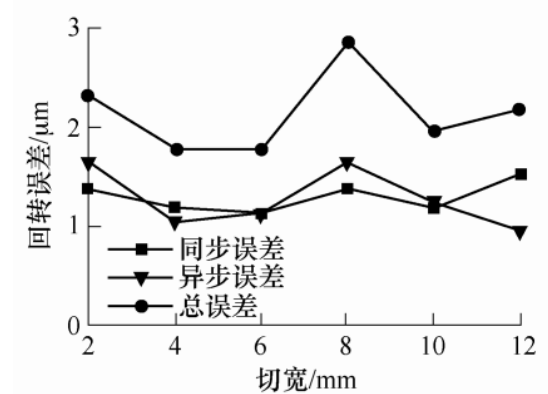

(b) 变切宽

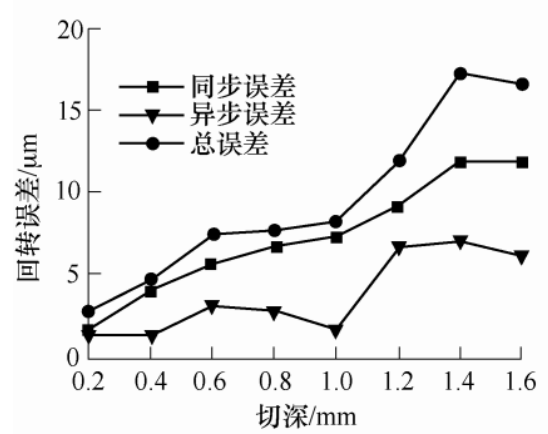

(c) 变切深

图 11 不同工况下回转精度计算结果

从图 11 中可以看到, 在变进给和变切深工况 下，同步误差随进给速度和切削深度的改变呈现有 规律的变化, 切深对同步误差影响最大, 进给速度 
次之。异步误差由于受到噪声的干扰呈无规律变化。 在变切宽情况下, 同步误差随切宽改变变化不大, 是 由于本试验中切宽的改变对切削力的影响不明显。

图 12 为测得的不同切深下 $X$ 方向的切削力时 域图和频谱。将不同切深下 $X$ 方向的切削力平均幅 值和回转精度值分别归一化。对比不同切深下的切 削力幅值和回转精度值归一化结果, 如图 13 所示。 可以看到, 同步误差随切深变化规律与切削力幅值 变化规律一致, 异步误差随机变化, 这正是只预测 同步误差的主要原因。同时, 图 13 反映了切削载荷 对主轴回转精度值具有极大的影响, 尤其体现为同 步误差随切削载荷增大而增大。这更加说明研究切 削工况下主轴回转精度问题才具有实际意义。

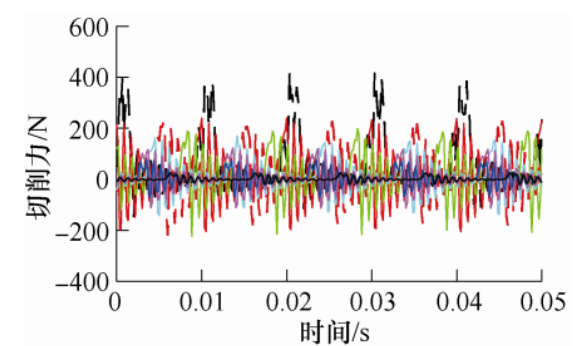

(a) 时域图

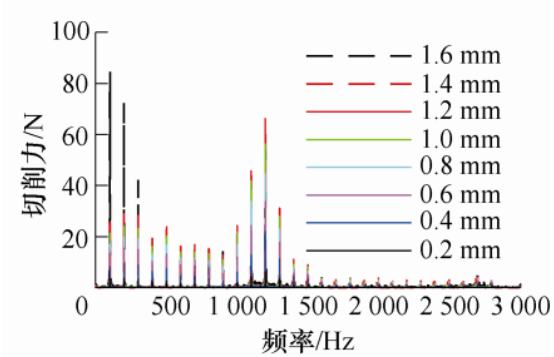

(b) 频谱图

图 12 不同切深下的切削力

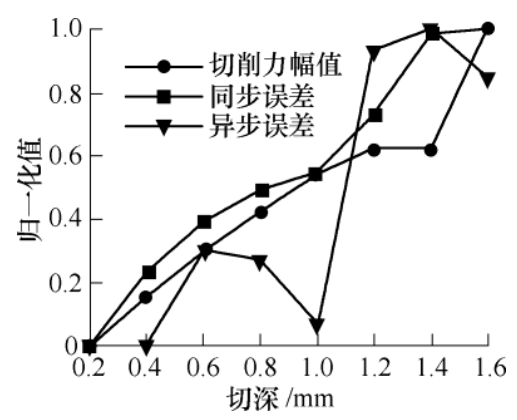

图 13 不同切深下切削力平均幅值与 回转精度值归一化结果

\section{2 结果对比与分析}

将上节测得的不同切削工况下的 $X 、 Y 、 Z$ 三个 方向的切削力信号经过修正后施加到建立的主轴动 力学模型刀尖位置处。图 14 为切宽 $2 \mathrm{~mm}$, 切深 $0.2 \mathrm{~mm}$, 进给速度 $500 \mathrm{~mm} / \mathrm{min}$ 时仿真得到的图 4 测点处振动位移响应。从图中可以看到, 切削工况
下的位移响应曲线较粗粘。

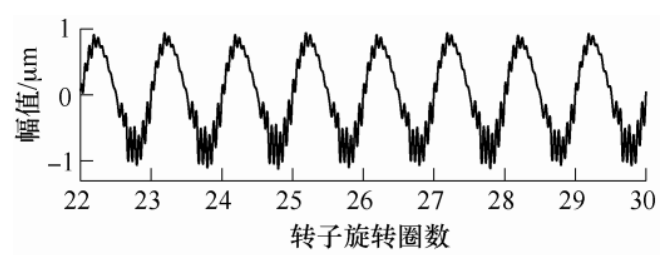

(a) $X$ 方向位移响应

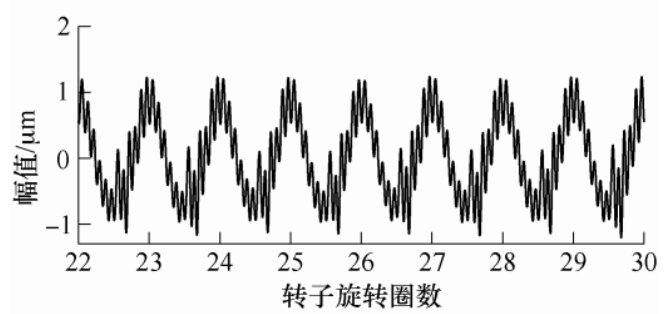

(b) $Y$ 方向位移响应

图 14 仿真振动位移响应结果

采用第 2 部分的回转精度计算和误差评定方法处 理不同工况下仿真得到的振动位移响应。图 15 为不 同工况下仿真同步误差与实测同步误差对比结果。

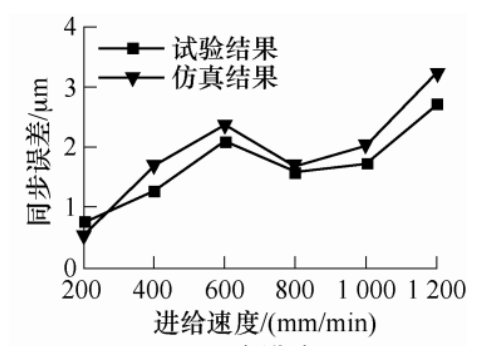

(a) 变进给

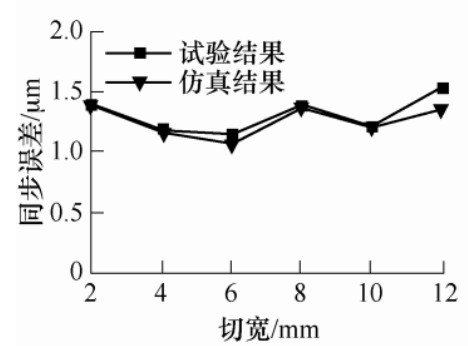

(b) 变切宽

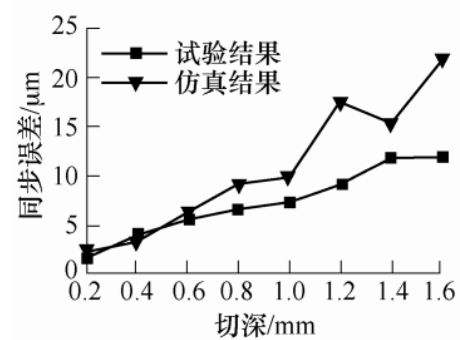

(c) 变切深

图 15 切削工况下仿真与试验对比结果

从图 15 中可以看到, 在变进给与变切宽工况下 试验与仿真吻合得很好, 最大误差在 $0.2 \mu \mathrm{m}$ 。在变 切深工况下, 切深较小时仿真与试验吻合很好, 但 是当切深较大时, 两者相差较大。这主要是由于切 
深较大时振动较大, 使得实际测得的位移信号中含 有较大噪声。噪声严重影响了误差分离的精度, 导 致分离出来的圆度误差不准确。

主轴在低速下空转时的噪声很小, 此时分离 出来的圆度误差可认为是主轴测量截面实际圆度 误差。因此, 将主轴 $6000 \mathrm{r} / \mathrm{min}$ 时切削工况下分 离出来的圆度误差与主轴 $100 \mathrm{r} / \mathrm{min}$ 空转分离得 到的圆度误差进行对比。图 16 为其中三种工况与 $100 \mathrm{r} / \mathrm{min}$ 圆度误差对比结果。从图中可以看到, 切宽 $12 \mathrm{~mm}$ 和进给速度 $1200 \mathrm{~mm} / \mathrm{min}$ 工况下分 离得到的圆度误差与主轴 $100 \mathrm{r} / \mathrm{min}$ 空转时得到 的圆度误差吻合的很好。而切深为 $1.2 \mathrm{~mm}$ 时分 离出来的圆度误差与 $100 \mathrm{r} / \mathrm{min}$ 空转时分离得到 的圆度误差差别较大, 这是因为大切深工况下测 量得到的位移信号的噪声较大。在误差分离时权 函数将噪声进一步放大, 使得得到的回转精度不 准确。
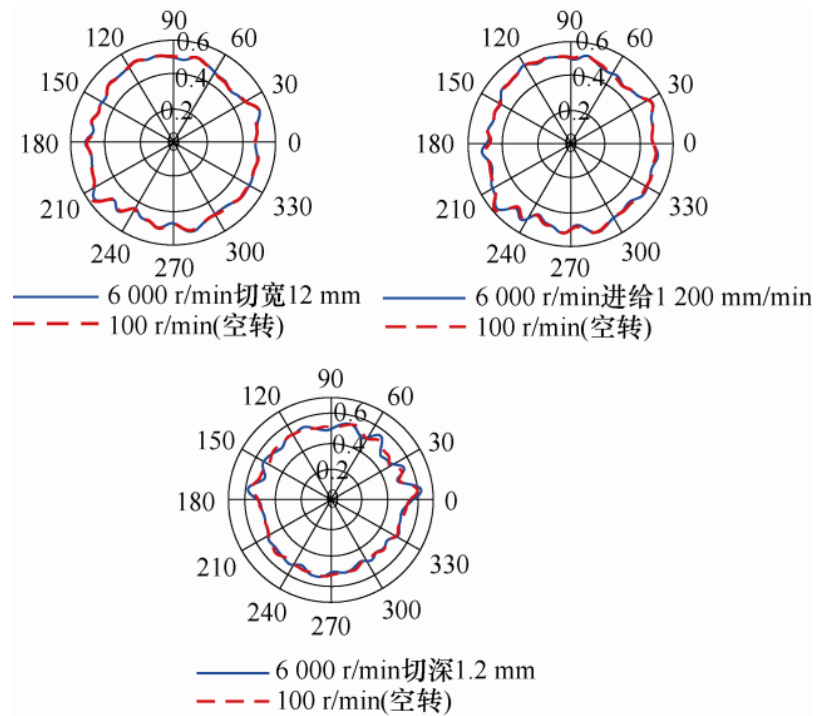

图 16 不同切削工况下圆度误差与 $100 \mathrm{r} / \mathrm{min}$ 空转时对比图

\section{4 结论}

(1) 基于高速主轴动力学模型, 提出了切削工 况下主轴动态回转精度预测方法。设计了一套无需 借助标准球的回转精度测试装置, 实现了切削工况 下的主轴回转精度测量。

(2) 得到了主轴回转误差随切削参数的变化 规律。在变进给和变切深工况下, 主轴同步误差 随进给速度和切削深度的增大而增大。在变切宽 工况下, 同步误差随切宽改变变化不大。在切削 工况下, 异步误差由于受到噪声的干扰呈无规律 变化。
(3) 仿真与试验对比结果表明, 提出的基于动 力学模型的主轴回转精度预测方法能够有效的预测 主轴在切削工况下的回转精度。

\section{参 考 文 献}

[1] ASHOK S D, SAMUEL G L. Modeling, measurement, and evaluation of spindle radial errors in a miniaturized machine tool[J]. The International Journal of Advanced Manufacturing Technology, 2012, 59(5): 445-461.

[2] AN C H, ZHANG Y, XU Q, et al. Modeling of dynamic characteristic of the aerostatic bearing spindle in an ultra-precision fly cutting machine[J]. International Journal of Machine Tools and Manufacture, 2010, 50(4): 374-385.

[3] 李树森, 刘噋. 精密离心机静压气体轴承主轴系统的动 力学特性分析 $[J]$. 机械工程学报, 2005, 41(2): 28-32. LI Shusen, LIU Tun. Analysis of the dynamics of precision centrifuge spindle system with the externally pressurized gas bearing[J]. Chinese Journal of Mechanical Engineering, 2005, 41(2): 28-32.

[4] 黄强, 于宗玲, 魏坤. 主轴回转精度的综合建模及仿真 分析方法[J]. 机床与液压, 2015，43(15): 146-150.

HUANG Qiang, YU Zongling, WEI Kun. General modeling and simulation analysis method on rotating accuracy of spindle[J]. Machine Tool \& Hydraulics, 2015, 43(15): 146-150.

[5] 熊万里, 侯志泉, 吕浪. 液体静压主轴回转误差的形成 机理研究[J]. 机械工程学报, 2014, 50(7): 112-119. XIONG Wanli, HOU Zhiquan, LÜ Lang. Study on the mechanism of hydrostatic spindle rotational error motion[J]. Journal of Mechanical Engineering, 2014, 50(7): 112-119.

[6] HUANG P, LEE W B, CHAN C Y. Investigation of the effects of spindle unbalance induced error motion on machining accuracy in ultra-precision diamond turning $[\mathrm{J}]$. International Journal of Machine Tools and Manufacture, 2015, 94: 48-56.

[7] MARTIN D L. Precision spindle and bearing error analysis[J]. International Journal of Machine Tools and Manufacture, 1995, 35(2): 187-193.

[8] KIM S H, KIM B H, JIN Y G. A study on the error separation method in rotation accuracy measurement of high precision spindle unit[J]. Journal of the Korean Society of Manufacturing Process Engineers, 2014, 13(1): $78-84$ 
[9] ANANDAN K P, OZDOGANLAR O B. A multiorientation error separation technique for spindle metrology of miniature ultra-high-speed spindles[J]. Precision Engineering, 2015, 43: 119-131.

[10] FUJIMAKI K, MITSUI K. Radial error measuring device based on auto-collimation for miniature ultra-high-speed spindles[J]. International Journal Machine Tool \& Manufacture, 2007, 47(11): 1677-1685.

[11] LIU Zichao, PAN Wei, LU Changhou, et al. Numerical analysis on the static performance of a new piezoelectric membrane restrictor[J]. Industrial Lubrication and Tribology, 2016, 68(5): 521-529.

[12] 杜正春, 杨建国, 姚振强, 等. 主轴回转误差补偿机理 和动力学模型研究 $[\mathrm{J}]$. 机械工程学报, 2003, 39(3): 48-57.

DU Zhengchun, YANG Jianguo, YAO Zhenqiang, et al. Research on mechanism and dynamics model of spindle rotation error compensation[J]. Journal of Mechanical Engineering, 2003, 39(3): 48-57.

[13] 胡灿, 熊万里, 孙文彪, 等. 可控节流液体静压主轴回 转精度提升的机理研究 [J]. 机械工程学报, 2019, 55(11): 160-168.

HU Can, XIONG Wanli, SUN Wenbiao, et al. Research on the mechanism of improving hydrostatic spindle rotating accuracy with controllable restrictor[J]. Journal of Mechanical Engineering, 2019， 55(11): 160-168.

[14] 陈恩平. 机床主轴回转精度计算机辅助测试系统 [J]. 组合机床与自动化加工技术，2004(9)：100-101.

CHEN Enping. The computer aided measuring system for spindle rotating accuracy measuring of machine tool[J]. Modular Machine Tool \& Automatic Manufacturing Technique, 2004(9): 100-101.

[15] 王卫东, 翟超, 陈柯. 机床主轴回转精度的 CCD 测量 系统[J]. 计量学报, 2006, 27(1): 18-21.

WANG Weidong, ZHAI Chao, CHEN Ke. A CCD measuring system for the rotary precision of spindle of machine tools[J]. Acta Metrologica Sinica, 2006, 27(1): 18-21.

[16] KARACAY T, AKTURK N. Vibrations of a grinding spindle supported by angular contact ball bearings[J]. Proceedings of the Institution of Mechanical Engineers Part K Journal of Multi-body Dynamics，2008，222(1): 61-75.

[17] KIM S M, LEE S K. Prediction of thermo-elastic behavior in a spindle-bearing system considering bearing surro-
undings[J]. International Journal of Machine Tools \& Manufacture, 2001, 41(6): 809-831.

[18] JIANG Shuyun, MAO Hebing. Investigation of variable optimum preload for a machine tool spindle[J]. International Journal of Machine Tools and Manufacture, 2010, 50 (1): 19-28.

[19] JIANG Shuyun, ZHENG Shufei. Dynamic design of a high-speed motorized spindle-bearing system[J]. Journal of Mechanical Design, 2010, 132 (3): 034501.

[20] JIANG Shuyun, ZHENG Shufei. A modeling approach for analysis and improvement of spindle-drawbar-bearing assembly dynamics [J]. International Journal of Machine Tools and Manufacture, 2010, 50 (1): 131-142.

[21] 陈小安, 张朋, 合烨, 等. 高速电主轴轴向振动研究 $[\mathrm{J}]$. 振动与冲击， 2014，33(20): 70-74.

CHEN Xiaoan, ZHANG Peng, HE Ye, et al. Axial vibration of high-speed motorized spindles[J]. Journal of Vibration and Shock，2014，33(20): 70-74.

[22] 陈小安, 刘俊峰, 合烨, 等. 高速电主轴热态性能及其 影响[J]. 机械工程学报, 2013，49(11): 135-142.

CHEN Xiaoan, LIU Junfeng, HE Ye, et al. Thermal properties of high speed motorized spindle and their effects[J]. Journal of Mechanical Engineering, 2013， 49 (11): 135-142.

[23] CAO Hongrui, ZHANG Xingwu, CHEN Xuefeng. The concept and progress of intelligent spindles: A review[J]. International Journal of Machine Tools \& Manufacture, 2017, 112: 112-115.

[24] XI Songtao, CAO Hongrui, CHEN Xuefeng. A dynamic modeling approach for spindle bearing system supported by both angular contact ball bearing and floating displacement bearing $[\mathrm{J}]$. Journal of Manufacturing Science and Engineering-Transactions of the ASME, 2018, 140(2): 021014.

[25] XI Songtao, CAO Hongrui, CHEN Xuefeng. Dynamic modeling of spindle bearing system and vibration response investigation[J]. Mechanical Systems and Signal Processing. 2019, 114: 486-511.

[26] 熊万里, 侯志泉, 吕浪, 等. 气体悬浮电主轴动态特性 研究进展 [J]. 机械工程学报, 2011，47(5): 40-58.

XIONG Wanli, HOU Zhiquan, LÜ Lang, et al. Review on the dynamic characteristics of aerostatic motorized spindles[J]. Journal of Mechanical Engineering, 2011, 47(5): $40-58$

[27] SHI Shengyu, LIN Jing, WANG Xiufeng, et al. A hybrid 
three-probe method for measuring the roundness error and the spindle error[J]. Precision Engineering, 2016, 45: 403-413.

[28] MA Ping, ZHAO Chunming, LU Xinhui. Rotation error measurement technology and experimentation research of high-precision hydrostatic spindle[J]. The International Journal of Advanced Manufacturing Technology, 2014,
73(9): 1313-1320.

作者简介: 康婷, 女, 1994 年出生。主要研究方向为机床主轴回转精度 分析。

E-mail: kt18829349535@stu.xjtu.edu.cn

曹宏瑞(通信作者), 男, 1982 年出生, 博士, 教授, 博士研究生导师。 主要研究方向为智能主轴振动监控基础理论与应用技术、机械结构有限 元分析、动力学建模与优化设计、机械设备非平稳信号处理方法及故障 诊断技术、航空发动机转子振动异常监测与故障预警方法。

E-mail: chr@mail.xjtu.edu 